\title{
Experimental Liquidus Studies of the $\mathrm{ZnO}-" \mathrm{CuO}_{0.5}$ " and $\mathrm{ZnO}$-“ $\mathrm{CuO}_{0.5}$ "-SiO 2 Liquidus in Equilibrium with $\mathrm{Cu}-\mathrm{Zn}$ Metal
}

\author{
M. Shevchenko ${ }^{1}$ (D) E. Jak ${ }^{1}$
}

Submitted: 6 March 2020/in revised form: 7 April 2020/Published online: 7 May 2020

(c) ASM International 2020

\begin{abstract}
Phase equilibria in the $\mathrm{ZnO}-$ " $\mathrm{CuO}_{0.5}$ "- $\mathrm{SiO}_{2}$ system have been investigated at 1403-1948 K (1130$1675^{\circ} \mathrm{C}$ ) for oxide liquid in equilibrium with $\mathrm{Cu}-\mathrm{Zn}$ metal $(>99 \% \mathrm{Cu})$ and solid oxide phases: (a) tridymite or cristobalite $\mathrm{SiO}_{2}$; (b) willemite $\mathrm{Zn}_{2} \mathrm{SiO}_{4}$; (c) zincite $\mathrm{ZnO}$; and (d) cuprite $\mathrm{Cu}_{2} \mathrm{O}$. Two-liquid immiscibility range in the high- $\mathrm{SiO}_{2}$ slags has also been studied. High-temperature equilibration on primary phase $\left(\mathrm{SiO}_{2}, \mathrm{Cu}_{2} \mathrm{O}\right)$, inert metal (platinum-iridium wire), or ceramic $\left(\mathrm{Al}_{2} \mathrm{O}_{3}\right.$ for high$\mathrm{Cu}_{2} \mathrm{O}$ slags) substrates, followed by quenching and direct measurement of $\mathrm{Zn}, \mathrm{Cu}, \mathrm{Si}$ and possible impurities (Al, Pt, Ir) concentrations in the phases with the electron probe $\mathrm{x}$-ray microanalysis (EPMA) has been used to accurately characterize the system in equilibrium with metal. All results are projected onto the $\mathrm{ZnO}-$ " $\mathrm{CuO}_{0.5}$ " $-\mathrm{SiO}_{2}$ plane for presentation purposes.
\end{abstract}

Keywords copper $\cdot$ phase diagrams $\cdot$ silica $\cdot$ slags $\cdot$ zinc

\section{Introduction}

The study of the $\mathrm{ZnO}$ - " $\mathrm{CuO}_{0.5}$ "- $\mathrm{SiO}_{2}$ system represents an important part of the overall integrated thermodynamic modelling and experimental research program for the multicomponent $\mathrm{Pb}-\mathrm{Zn}-\mathrm{Fe}-\mathrm{Cu}-\mathrm{Si}-\mathrm{Ca}-\mathrm{Al}-\mathrm{Mg}-\mathrm{O}$ system. Its study provides essential thermodynamic information to predict distribution of $\mathrm{Cu}$ in $\mathrm{ZnO}$-rich slags and $\mathrm{Zn}$ in

M. Shevchenko

m.shevchenko@uq.edu.au

1 Pyrometallurgy Innovation Centre (PYROSEARCH), The University of Queensland, Brisbane, QLD 4072, Australia
$\mathrm{Cu}_{2} \mathrm{O}$-rich slags, the importance of this is increasing due to development of pyrometallurgical recycling of complex secondary materials, such as waste electrical and electronic equipment (WEEE), and other common materials containing brass.

A preliminary study of the liquidus surface of the $\mathrm{ZnO}$ $\mathrm{CuO}_{0.5}-\mathrm{CuO}-\mathrm{SiO}_{2}$ system has been carried out by Xia and Taskinen. ${ }^{[1-3]}$ Most of the data in that study were obtained in air, which is far from the pyrometallurgical processing conditions; only two points at $1200{ }^{\circ} \mathrm{C}$ have been obtained in the ternary region in equilibrium with metal. No other studies have been found in literature.

\section{Experimental Technique and Procedure}

The experimental procedure and apparatus have been described in detail in previous publications by the authors. ${ }^{[4-6]}$ The initial mixtures were prepared by mixing high-purity powders $\mathrm{Cu}_{2} \mathrm{O}$ ( 99.9 wt.\% purity) supplied by Heysham, Great Britain, and $\mathrm{ZnO}$ (99.8 wt.\% purity), $\mathrm{SiO}_{2}$ (99.9 wt.\% purity), and $\mathrm{Cu}$ ( 99.9 wt.\% purity) supplied by

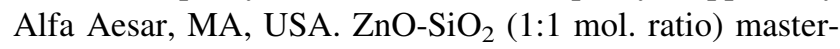
slag was used in several open substrate experiments to reduce evaporation loss of $\mathrm{ZnO}$. The mixtures were pressed into pellets using a tool steel die. Less than $0.5 \mathrm{~g}$ of mixture was used in each equilibration experiment. The initial compositions of the mixtures were selected so that one or more crystalline phases would be present in equilibrium with liquid slag and approximately 10 vol. $\%$ metal. The volume fraction of solids was targeted to be below 50 vol.\%, and preferably about $10 \mathrm{vol} \%$, to achieve rapid equilibration and useful outcomes from quenching (it was found that the solids serve as heterogeneous nucleation centers, and the minimum distance between them should 
exceed 10-20 micron to ensure that amorphous slag of uniform composition, unaffected by dendritic crystal growth, is obtained). An iterative procedure involving preliminary experiments was often needed to achieve the targeted proportions of the phases for a given final temperature and composition.

Four types of substrates were used for equilibration, depending on the conditions:

1. Silica ampoules $(5 \mathrm{~cm}$ long, $1.3 \mathrm{~cm}$ outer diameter, $0.1 \mathrm{~cm}$ thick wall, $99.999 \% \mathrm{SiO}_{2}$, supplied by GY, Jiangsu, China) sealed under vacuum ( $0.001 \mathrm{~atm})$, or with small holes, or open silica crucibles for the samples in the cristobalite/tridymite primary phase fields. Sealing allowed preventing the loss of the volatile $\mathrm{Zn}$ from the system, as well as oxidation of the samples by residual oxygen in the gas atmosphere. Pelletizing of the powder mixture was used to avoid the presence of loose powder particles, which could be preferentially extracted from the ampoule during evacuation. The protection against $\mathrm{Zn}$ evaporation is increased in the order open crucible $<$ ampoule with hole $<$ sealed ampoule. However, the quenching speed decreases in the same order. Experiments on open substrates were limited to low temperatures (up to $1400{ }^{\circ} \mathrm{C}$ ), because at higher temperatures the loss of $\mathrm{Zn}$ becomes too fast to allow reasonable equilibration time.

2. Willemite $\left(\mathrm{Zn}_{2} \mathrm{SiO}_{4}\right)$-covered $\mathrm{SiO}_{2}$ crucibles-prepared by melting $\mathrm{ZnO}-\mathrm{SiO}_{2}$ mixture on the $\mathrm{SiO}_{2}$ substrate. However, willemite layer was usually not dense enough to prevent significant diffusion of $\mathrm{SiO}_{2}$ from crucible to slag, so this assemblage did not work for low- $\mathrm{SiO}_{2}$ ranges. Direct diffusion through willemite solid layer is considered unlikely. Possible diffusion is due to cracks in the willemite layer. Since they were not found in the given experiments, and the liquid composition was measured at variable distances from the substrate and found to be homogeneous, these experiments were included as successful.

3. $\mathrm{Al}_{2} \mathrm{O}_{3}$ crucibles $(99.7 \%, 8 \mathrm{~mm}$ inner diameter, $15 \mathrm{~mm}$ height, supplied by Xiamen Wintrustek, Fujian, China) were used only in the range within or close to the $\mathrm{ZnO}$ $\mathrm{Cu}_{2} \mathrm{O}$ subsystem, since it was found that high- $\mathrm{Cu}_{2} \mathrm{O}$, low- $\mathrm{SiO}_{2}$ slags have low solubility of $\mathrm{Al}_{2} \mathrm{O}_{3}(\sim 1 \%)$, while difficult to handle on other substrates due to high fluidity and fast loss of $\mathrm{Zn}$. The layer of $\mathrm{ZnAl}_{2} \mathrm{O}_{4}$ was found to be dense with no cracks. No detached $\mathrm{ZnAl}_{2} \mathrm{O}_{4}$ particles were present. Also, liquid and zincite measured at variable distances from the substrate were found to have the same composition. This indicates that liquid and zincite have reached saturation with $\mathrm{Al}_{2} \mathrm{O}_{3}$ within the first minutes during formation of $\mathrm{ZnAl}_{2} \mathrm{O}_{4}$, and further diffusion is likely to be slow.

4. Iridium $(99.99 \%)$ or platinum-30\% iridium wire (supplied by Huanya, Jiangsu, China) substrate was used for the samples in the willemite, zincite and cuprite primary phase fields. Contamination of $\mathrm{Cu}$ metal by platinum (up to $30 \%$ ) and iridium (up to $3 \%$ ), and probability of the wire breaking are the limitations of this substrate type. As soon as there is $>70 \mathrm{wt} . \% \mathrm{Cu}$ in metal ( $>90$ mol.\% since the impurities Ir and Pt are heavy), the effect on phase equilibria due to formation of $\mathrm{Cu}^{2+}$ in slag can be neglected.

All equilibration experiments were carried out in a vertical reaction tube (impervious recrystallized alumina, 30-mm inner diameter) within an electrical resistance ( $\mathrm{SiC}$ or $\mathrm{MoSi}_{2}$ ) heated furnace. The samples were placed immediately adjacent to a working thermocouple in a recrystallized alumina sheath in the uniform hot zone of the furnace to monitor the actual sample temperature. The working thermocouple was calibrated against a standard thermocouple (supplied by the National Measurement Institute of Australia, NSW, Australia). The overall absolute temperature accuracy of the experiment was estimated to be $\pm 3 \mathrm{~K}$.

The samples were suspended in the hot zone of the furnace by Kanthal support wire (Fe-Cr-Al alloy, 0.7 or 1-mm diameter). For temperatures higher than $1773 \mathrm{~K}$ $\left(1500{ }^{\circ} \mathrm{C}\right)$, a $20-25 \mathrm{~cm}$ Mo or Pt-Rh wire was used to extend the support, so that the Kanthal wire is not in the hot zone. The samples were pre-melted for 5-10 min at 20-100 $\mathrm{K}$ above the equilibration temperature, to form a homogeneous liquid slag. Some samples in the ranges where even a small additional increase in temperature could cause failure due to evaporation of $\mathrm{Zn}$ or broken substrate were not premelted. Premelt was followed by equilibration at the final target temperature for the required time. A compromise had to be found between longer time for better equilibration and the risk to lose the volatile component or destroy the substrate. The analysis of various parts of the samples did not indicate significant non-equilibrium. Shorter times were limited to low- $\mathrm{SiO}_{2}$ (low viscosity) or high-temperature (high reaction rate) ranges. At the end of the equilibration process, the samples were released and rapidly quenched into the $\mathrm{CaCl}_{2}$ brine $\left(<253 \mathrm{~K}\left(-20^{\circ} \mathrm{C}\right)\right)$. The samples were then washed thoroughly in water and ethanol before being dried on a hot plate, mounted in epoxy resin, and cross-sectioned using conventional metallographic polishing techniques.

The samples were examined by optical microscopy, carbon-coated, and the phase compositions were measured using an electron probe $\mathrm{x}$-ray microanalysis technique with wavelength dispersive detectors (JEOL 8200L EPMA; 
Japan Electron Optics Ltd., Tokyo, Japan). The EPMA was operated with $15 \mathrm{kV}$ accelerating voltage and $20 \mathrm{nA}$ probe current. Copper $(\mathrm{Cu})$, quartz $\left(\mathrm{SiO}_{2}\right)$, wollastonite $\left(\mathrm{CaSiO}_{3}\right)$, corundum $\left(\mathrm{Al}_{2} \mathrm{O}_{3}\right)$ (supplied by Charles M. Taylor Co., Stanford, CA), and zincite (sintered from $99.8 \% \mathrm{ZnO}$ powder) standards were used for calibration of EPMA. The concentrations of metal cations were measured with EPMA; no information on the oxidation states of the metal cations was obtained. Copper oxide concentrations were recalculated as $\mathrm{Cu}_{2} \mathrm{O}$ for presentation purposes only; this oxidation state was selected according to the information on the slags in equilibrium with metal in the $\mathrm{Cu}-\mathrm{Si}-\mathrm{O}$ system. ${ }^{[7]}$ The molar ratios of the oxides were used in the graphical presentation of the data.

The standard Duncumb-Philibert atomic number, absorption, and fluorescence (ZAF) correction supplied by $\mathrm{JEOL}^{[8-10]}$ was used. The standard ZAF correction was further improved following an approach similar to the one previously described. ${ }^{[11-13]}$ The accuracy of ZAF correction for the " $\mathrm{CuO}_{0.5}$ "- $\mathrm{SiO}_{2}$ system has been checked recently, ${ }^{[14]}$ using dehydrated natural dioptase $\left(\mathrm{CuSiO}_{3}\right)$ as a reference material of fixed composition; overestimation of $\mathrm{SiO}_{2}$ concentration by $1.8 \%$ was observed. In the $\mathrm{ZnO}-$ $\mathrm{SiO}_{2}$ system, willemite $\mathrm{Zn}_{2} \mathrm{SiO}_{4}$ was used as a reference material. ${ }^{[15,16]}$ The combined correction formulas for the $\mathrm{ZnO}$ - "CuO $\mathrm{Cu}_{0.5}$ "- $\mathrm{SiO}_{2}$ slags are:

$x_{\mathrm{SiO}_{2}}^{\text {corrected }}=x_{\mathrm{SiO}_{2}}-0.07 \cdot x_{\mathrm{SiO}_{2}} x_{\mathrm{CuO}_{0.5}}-0.07 \cdot x_{\mathrm{SiO}_{2}} x_{\mathrm{ZnO}}$

$x_{\mathrm{CuO}_{0.5}}^{\text {corrected }}=x_{\mathrm{CuO}_{0.5}}+0.07 \cdot x_{\mathrm{SiO}_{2}} x_{\mathrm{CuO}_{0.5}}$

$x_{\mathrm{ZnO}}^{\text {corrected }}=x_{\mathrm{ZnO}}+0.07 \cdot x_{\mathrm{SiO}_{2}} x_{\mathrm{ZnO}}$

Same correction is used for previous data obtained in the " $\mathrm{CuO}_{0.5}$ " $-\mathrm{SiO}_{2}$ system. ${ }^{[17]}$

The ability to quench the liquid slag phase to ambient temperature without the onset of crystallization was found to depend on the composition of the slag and the equilibration temperature. Significant problems were observed with low-silica slags in the zincite primary phase field, where the glassy homogeneous areas were only a small proportion of the melt, believed to be formed at the surfaces directly contacting the quenching medium. For these samples the approach taken to obtain accurate, repeatable, and objective measurements of the average compositions of the liquid slag phase by the use of EPMA was similar to that described by Nikolic et al. ${ }^{[18]}$ : an average of at least 20 points in the best quenched area (usually, near the surface) was used, additionally controlled by standard deviation of composition not exceeding $1 \mathrm{~mol} \%$. In cases where melt crystallization was rapid, the experiments were repeated until an area of sufficiently well-quenched microstructure was found. Increasing the probe diameter to 20-50 micron resulted in reduced variability of the measured liquid slag compositions, while zero probe diameter was only used for solid phases.

Special experiments were undertaken in the present study with the 50-200 micron size grains of pure unreacted quartz pressed together with the $\mathrm{Cu}$ and $\mathrm{ZnO}$ powders, and crushed sintered zincite with the $\mathrm{Cu}$ powder at room temperature (unreacted couples). Apparent 1.05 mol.\% " $\mathrm{CuO}_{0.5}$ " and $0.82 \mathrm{~mol} \% \mathrm{ZnO}$ are observed due to the secondary $\mathrm{x}$-ray fluorescence in pure quartz grains surrounded by $\mathrm{Cu}$ and $\mathrm{ZnO}$, respectively. Apparent 1.03 mol.\% " $\mathrm{CuO}_{0.5}$ " is observed in pure zincite grains surrounded by $\mathrm{Cu}$. It was found in the previous studies of other systems involving elements with high-energy $(>5 \mathrm{keV})$ lines $(\mathrm{Fe}, \mathrm{Cu}, \mathrm{Zn})^{[14,16,19]}$ that the secondary fluorescence effect can be observed over large distances ( $>100$ micron). This is much more than the typical size of the observed solid particles in the present system (10-40 micron). Therefore, the $\mathrm{x}$-rays formed in these particles have low probability not to exit them into the surrounding slag matrix. This allows to assume that the geometry effect was comparable in all observed cases. The following correction for the secondary $\mathrm{x}$-ray fluorescence (similar to $\left.{ }^{[14,16,19]}\right)$ therefore has been developed and applied in the present study to the compositions of tridymite and cristobalite measured by EPMA:

$$
\begin{gathered}
x\left(\mathrm{CuO}_{0.5} \text { in } \mathrm{SiO}_{2}\right)^{\mathrm{corr}}=x\left(\mathrm{CuO}_{0.5} \text { in } \mathrm{SiO}_{2}\right)^{\text {initial }} \\
-0.0093 * \text { wt. fraction }\left(\mathrm{CuO}_{0.5} \text { in slag }\right), \\
x\left(\mathrm{ZnO} \text { in } \mathrm{SiO}_{2}\right)^{\text {corr }}=x\left(\mathrm{ZnO} \text { in } \mathrm{SiO}_{2}\right)^{\text {initial }} \\
-0.0082 \text { wt. fraction }(\mathrm{ZnO} \text { in slag }) ;
\end{gathered}
$$

for zincite:

$$
\begin{aligned}
x\left(\mathrm{CuO}_{0.5} \text { in } \mathrm{ZnO}\right)^{\text {corr }}= & x\left(\mathrm{CuO}_{0.5} \text { in } \mathrm{ZnO}\right)^{\text {initial }}-0.0092 \\
& * \text { wt. fraction }\left(\mathrm{CuO}_{0.5} \text { in slag }\right),
\end{aligned}
$$

for willemite:

$$
\begin{aligned}
x\left(\mathrm{CuO}_{0.5} \text { in } \mathrm{Zn}_{2} \mathrm{SiO}_{4}\right)^{\text {corr }}= & x\left(\mathrm{CuO}_{0.5} \text { in } \mathrm{Zn}_{2} \mathrm{SiO}_{4}\right)^{\text {initial }}-0.0092 \\
& * \text { wt. fraction }\left(\mathrm{CuO}_{0.5} \text { in slag }\right) .
\end{aligned}
$$

According to these formulae, most of observed solubility of $\mathrm{ZnO}$ in tridymite and cristobalite, and " $\mathrm{CuO}_{0.5}$ " in tridymite, cristobalite and willemite are due to the effect of secondary x-ray fluorescence. However, only part of observed " $\mathrm{CuO}_{0.5}$ " in zincite can be attributed to fluorescence, while most of it is a real solubility.

The effect of secondary fluorescence on slag composition measurement is mostly accounted by the corrections using willemite and dioptase reference standards. One exception is that fake increase of $\mathrm{Cu}$ in slag can be found near large $\mathrm{Cu}$ particles-these locations were avoided during analysis. 


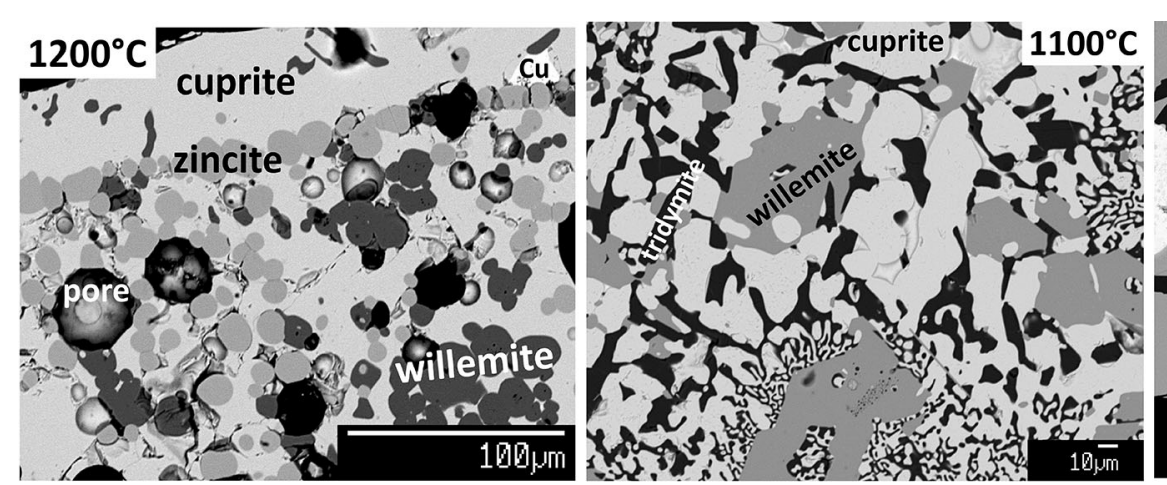

(a)

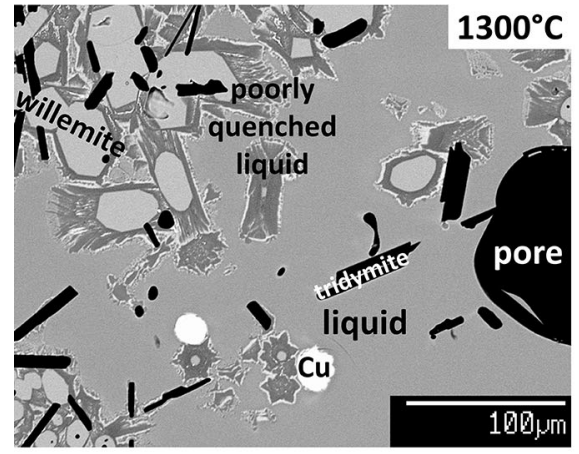

(d) (b)

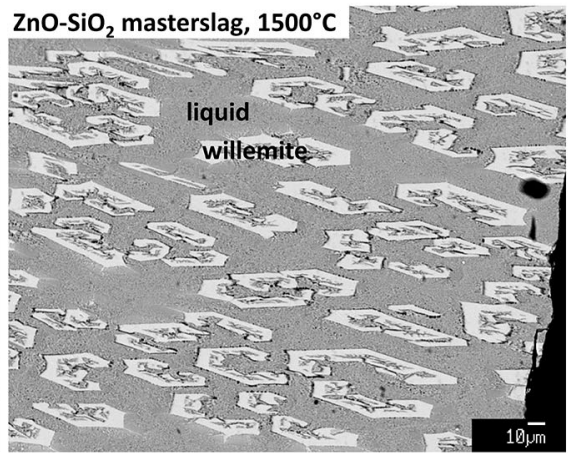

(e)

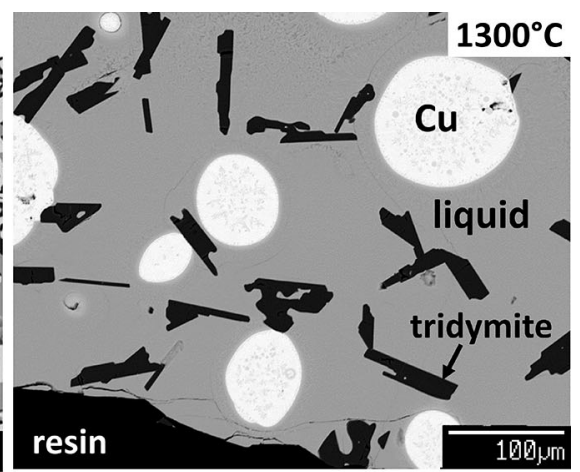

(c)

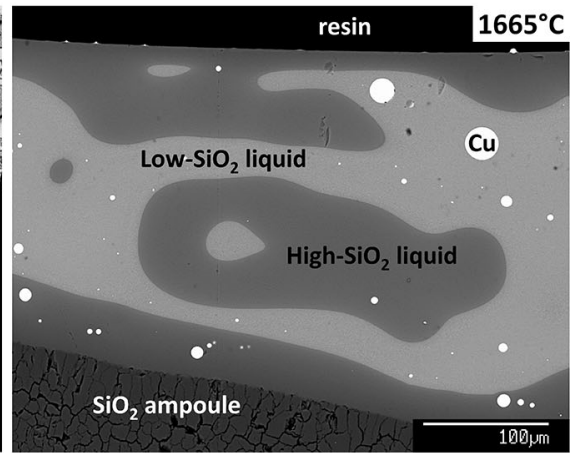

(f)

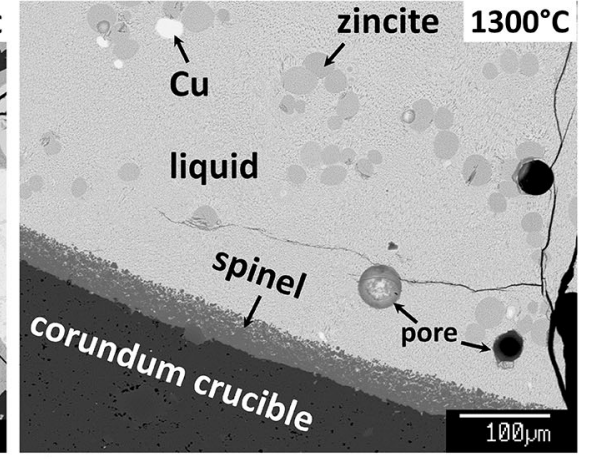

(h)

dendritic growth $)+$ tridymite $\left(\mathrm{SiO}_{2}\right)+$ willemite $\left(\mathrm{Zn}_{2} \mathrm{SiO}_{4}\right)+\mathrm{Cu}$, (e) Masterslag (molten $\mathrm{ZnO}+\mathrm{SiO}_{2}$ mixture), with willemite precipitation, (f) Two immiscible liquids $+\mathrm{Cu}$, (g) Liquid + cuprite $\left(\mathrm{Cu}_{2} \mathrm{O}\right)+$ tridymite $\left(\mathrm{SiO}_{2}\right)+\mathrm{Cu}, \quad$ (h) Liquid + zincite $(\mathrm{ZnO})+$ $\mathrm{Cu}+$ spinel $\left(\mathrm{ZnAl}_{2} \mathrm{O}_{4}\right)$ on corundum $\left(\mathrm{Al}_{2} \mathrm{O}_{3}\right)$

and steeply rising $\mathrm{ZnO}$ liquidus $(\mathrm{ZnO}$ in slag does not increase significantly by $1400{ }^{\circ} \mathrm{C}$ ), indicating strong positive deviations from ideal solution behaviour (Fig. 3). In the present study, $\mathrm{Al}_{2} \mathrm{O}_{3}$ dissolves in both liquid and zincite, and is expected to stabilize both approximately equally. We acknowledge that this is not true $\mathrm{Cu}_{2} \mathrm{O}-\mathrm{ZnO}-$ $\mathrm{SiO}_{2}$ system, but only the closest approximation of it achieved so far (in this problematic range of compositions). Plotted points are projections. Two immiscible liquids formation in the central range of the " $\mathrm{CuO}_{0.5}$ "- $\mathrm{ZnO}$ system 
Table 1 Experimental points on the liquidus surface with compositions of phases in the $\mathrm{ZnO}$-" $\mathrm{CuO}_{0.5}$ - $-\mathrm{SiO}_{2}$ system in equilibrium with metal

\begin{tabular}{|c|c|c|c|c|c|c|c|c|}
\hline \multirow[t]{2}{*}{ No. } & \multirow[t]{2}{*}{$T,{ }^{\circ} \mathrm{C}$} & \multirow[t]{2}{*}{ Substrate } & \multirow[t]{2}{*}{ Premelt $T,{ }^{\circ} \mathrm{C}$} & \multirow[t]{2}{*}{ Time, h } & \multirow[t]{2}{*}{ Phase } & \multicolumn{3}{|c|}{ Composition, mol.\% } \\
\hline & & & & & & $\mathrm{SiO}_{2}$ & $\mathrm{ZnO} / \mathrm{Zn}$ & $\mathrm{CuO}_{0.5} / \mathrm{Cu}$ \\
\hline \multicolumn{9}{|c|}{ Tridymite and cristobalite $\left(\mathrm{SiO}_{2}\right)$ liquidus } \\
\hline \multirow[t]{2}{*}{1} & 1140 & $\mathrm{SiO}_{2} \mathrm{amp}$ & 1240 & $0.25+18$ & Liquid & $24.7 \pm 0.8$ & $9.5 \pm 1.3$ & $65.8 \pm 2.0$ \\
\hline & & & & & Tridymite & $99.6 \pm 0.13$ & $0.13 \pm 0.06$ & $0.26 \pm 0.09$ \\
\hline \multirow[t]{2}{*}{2} & 1150 & $\mathrm{SiO}_{2} \mathrm{amp}+$ hole & 1240 & $0.25+2$ & Liquid & $23.9 \pm 0.11$ & $9.1 \pm 0.3$ & $67.1 \pm 0.3$ \\
\hline & & & & & Tridymite & $99.7 \pm 0.17$ & $0.11 \pm 0.05$ & $0.23 \pm 0.14$ \\
\hline \multirow[t]{2}{*}{3} & 1200 & $\mathrm{SiO}_{2}$ amp + hole & 1240 & $0.25+2$ & Liquid & $24.1 \pm 0.2$ & $8.0 \pm 0.3$ & $67.9 \pm 0.4$ \\
\hline & & & & & Tridymite & $99.5 \pm 0.16$ & $0.12 \pm 0.05$ & $0.35 \pm 0.14$ \\
\hline \multirow[t]{2}{*}{4} & 1200 & $\mathrm{SiO}_{2}$ open crucible & 1250 & $0.25+1.5$ & Liquid & $34.6 \pm 0.13$ & $19.4 \pm 0.4$ & $46.0 \pm 0.4$ \\
\hline & & & & & Tridymite & $99.3 \pm 0.1$ & $0.65 \pm 0.12$ & $0.08 \pm 0.02$ \\
\hline \multirow[t]{2}{*}{5} & 1200 & $\mathrm{SiO}_{2}$ open crucible & 1250 & $0.25+1.5$ & Liquid & $31.7 \pm 0.3$ & $15.7 \pm 0.5$ & $52.6 \pm 0.7$ \\
\hline & & & & & Tridymite & $99.5 \pm 0.1$ & $0.26 \pm 0.04$ & $0.25 \pm 0.07$ \\
\hline \multirow[t]{2}{*}{6} & 1200 & $\mathrm{SiO}_{2}$ open crucible & 1250 & $0.25+1.5$ & Liquid & $32.2 \pm 0.2$ & $17.7 \pm 0.5$ & $50.1 \pm 0.6$ \\
\hline & & & & & Tridymite & $99.5 \pm 0.1$ & $0.26 \pm 0.04$ & $0.25 \pm 0.07$ \\
\hline \multirow[t]{2}{*}{7} & 1300 & $\mathrm{SiO}_{2}$ open crucible & 1320 & $0.25+1.5$ & Liquid & $42.3 \pm 0.16$ & $31.3 \pm 0.4$ & $26.3 \pm 0.5$ \\
\hline & & & & & Tridymite & $99.1 \pm 0.12$ & $0.8 \pm 0.1$ & $0.1 \pm 0.08$ \\
\hline \multirow[t]{2}{*}{8} & 1300 & $\mathrm{SiO}_{2}$ open crucible & 1320 & $0.25+1.5$ & Liquid & $30.4 \pm 0.17$ & $11.0 \pm 0.3$ & $58.6 \pm 0.4$ \\
\hline & & & & & Tridymite & $99.6 \pm 0.2$ & $0.14 \pm 0.02$ & $0.28 \pm 0.2$ \\
\hline \multirow[t]{2}{*}{9} & 1300 & $\mathrm{SiO}_{2}$ open crucible & 1320 & $0.25+1.5$ & Liquid & $35.5 \pm 0.3$ & $17.2 \pm 0.5$ & $47.3 \pm 0.8$ \\
\hline & & & & & Tridymite & $99.4 \pm 0.17$ & $0.34 \pm 0.08$ & $0.3 \pm 0.09$ \\
\hline \multirow[t]{3}{*}{10} & 1300 & $\mathrm{SiO}_{2}$ open crucible & 1320 & $0.25+1.5$ & Liquid & $37.6 \pm 0.5$ & $21.1 \pm 0.5$ & $41.3 \pm 0.8$ \\
\hline & & & & & Tridymite & $99.4 \pm 0.17$ & $0.34 \pm 0.08$ & $0.3 \pm 0.09$ \\
\hline & & & & & Metal & 0 & $0.14 \pm 0.02$ & $99.86 \pm 0.02$ \\
\hline 11 & 1330 & $\mathrm{SiO}_{2} \mathrm{amp}+$ hole & No & 2 & Liquid & $30.0 \pm 0.5$ & $7.8 \pm 0.7$ & $62.2 \pm 1.0$ \\
\hline & & & & & Tridymite & $99.5 \pm 0.03$ & $0.16 \pm 0.03$ & $0.3 \pm 0.03$ \\
\hline 12 & 1350 & $\mathrm{SiO}_{2}$ open crucible & 1380 & $0.25+1.5$ & Liquid & $44.9 \pm 0.16$ & $35.0 \pm 0.3$ & $20.1 \pm 0.2$ \\
\hline & & & & & Tridymite & $99.2 \pm 0.11$ & $0.7 \pm 0.04$ & $0.12 \pm 0.07$ \\
\hline 13 & 1350 & $\mathrm{SiO}_{2}$ open crucible & 1380 & $0.25+1.5$ & Liquid & $44.8 \pm 0.06$ & $36.7 \pm 0.13$ & $18.5 \pm 0.13$ \\
\hline & & & & & Tridymite & $99.2 \pm 0.11$ & $0.7 \pm 0.04$ & $0.12 \pm 0.07$ \\
\hline & & & & & Metal & 0 & $0.13 \pm 0.08$ & $99.87 \pm 0.08$ \\
\hline 14 & 1400 & $\mathrm{SiO}_{2}$ open crucible & 1410 & $0.25+1.5$ & Liquid & $34.0 \pm 0.2$ & $10.1 \pm 0.4$ & $55.9 \pm 0.3$ \\
\hline & & & & & Tridymite & $99.6 \pm 0.2$ & $0.13 \pm 0.06$ & $0.23 \pm 0.15$ \\
\hline 15 & 1400 & $\mathrm{SiO}_{2}$ open crucible & 1410 & $0.25+1.5$ & Liquid & $40.5 \pm 0.5$ & $18.4 \pm 0.8$ & $41.1 \pm 1.2$ \\
\hline & & & & & Tridymite & $99.4 \pm 0.08$ & $0.3 \pm 0.06$ & $0.26 \pm 0.06$ \\
\hline 16 & 1400 & $\mathrm{SiO}_{2} \mathrm{amp}$ & 1420 & $0.2+3$ & Liquid & $46.6 \pm 0.14$ & $42.9 \pm 0.14$ & $10.5 \pm 0.11$ \\
\hline & & & & & Tridymite & $99.0 \pm 0.02$ & $0.7 \pm 0.2$ & $0.27 \pm 0.2$ \\
\hline & & & & & Metal & 0 & $0.28 \pm 0.14$ & $99.72 \pm 0.14$ \\
\hline 17 & 1500 & $\mathrm{SiO}_{2} \mathrm{amp}$ & 1520 & $0.2+2$ & Liquid & $24.1 \pm 0.4$ & 0 & $75.9 \pm 0.4$ \\
\hline & & & & & Cristobalite & 99.7 & 0 & 0.3 \\
\hline 18 & 1500 & $\mathrm{SiO}_{2} \mathrm{amp}$ & 1520 & $0.2+2$ & Liquid & $42.2 \pm 0.2$ & $12.2 \pm 0.2$ & $45.6 \pm 0.3$ \\
\hline & & & & & Cristobalite & $99.4 \pm 0.03$ & $0.2 \pm 0.01$ & $0.4 \pm 0.02$ \\
\hline 19 & 1500 & $\mathrm{SiO}_{2} \mathrm{amp}$ & No & 1 & Liquid & $50.5 \pm 0.13$ & $24.6 \pm 0.15$ & $24.9 \pm 0.17$ \\
\hline & & & & & Cristobalite & $99.0 \pm 0.06$ & $0.5 \pm 0.06$ & $0.5 \pm 0.03$ \\
\hline 20 & 1500 & $\mathrm{SiO}_{2} \mathrm{amp}$ & 1520 & $0.2+2$ & Liquid & $50.3 \pm 0.16$ & $34.7 \pm 0.18$ & $15.0 \pm 0.12$ \\
\hline & & & & & Cristobalite & $99.2 \pm 0.02$ & $0.6 \pm 0.08$ & $0.2 \pm 0.06$ \\
\hline & & & & & Metal & 0 & $0.25 \pm 0.06$ & $99.75 \pm 0.06$ \\
\hline 21 & 1550 & $\mathrm{SiO}_{2}$ open crucible & 1570 & $0.2+1$ & Liquid & $27.7 \pm 0.4$ & 0 & $72.3 \pm 0.4$ \\
\hline & & & & & Cristobalite & 99.7 & 0 & 0.3 \\
\hline 22 & 1600 & $\mathrm{SiO}_{2} \mathrm{amp}$ & No & 1 & Liquid & $32.5 \pm 0.6$ & 0 & $67.5 \pm 0.6$ \\
\hline & & & & & Cristobalite & 99.7 & 0 & 0.3 \\
\hline 23 & 1600 & $\mathrm{SiO}_{2} \mathrm{amp}$ & No & 1 & Liquid & $39.9 \pm 0.5$ & $4.6 \pm 0.12$ & $55.5 \pm 0.4$ \\
\hline & & & & & Cristobalite & 99.5 & 0.1 & 0.4 \\
\hline
\end{tabular}


Table 1 continued

\begin{tabular}{|c|c|c|c|c|c|c|c|c|}
\hline \multirow[t]{2}{*}{ No. } & \multirow[t]{2}{*}{$T,{ }^{\circ} \mathrm{C}$} & \multirow[t]{2}{*}{ Substrate } & \multirow[t]{2}{*}{ Premelt $T,{ }^{\circ} \mathrm{C}$} & \multirow[t]{2}{*}{ Time, $\mathrm{h}$} & \multirow[t]{2}{*}{ Phase } & \multicolumn{3}{|c|}{ Composition, mol.\% } \\
\hline & & & & & & $\mathrm{SiO}_{2}$ & $\mathrm{ZnO} / \mathrm{Zn}$ & $\mathrm{CuO}_{0.5} / \mathrm{Cu}$ \\
\hline \multirow[t]{2}{*}{24} & 1600 & $\mathrm{SiO}_{2} \mathrm{amp}$ & No & 1 & Liquid & $51.3 \pm 0.1$ & $12.1 \pm 0.1$ & $36.6 \pm 0.16$ \\
\hline & & & & & Cristobalite & 99.4 & 0.3 & 0.3 \\
\hline \multirow[t]{2}{*}{25} & 1600 & $\mathrm{SiO}_{2} \mathrm{amp}$ & No & 1 & Liquid & $55.3 \pm 0.15$ & $17.2 \pm 0.14$ & $27.4 \pm 0.2$ \\
\hline & & & & & Cristobalite & $99.3 \pm 0.13$ & $0.4 \pm 0.09$ & $0.3 \pm 0.06$ \\
\hline \multirow[t]{3}{*}{26} & 1600 & $\mathrm{SiO}_{2} \mathrm{amp}$ & No & 1 & Liquid & $57.2 \pm 0.3$ & $29.2 \pm 0.16$ & $13.6 \pm 0.15$ \\
\hline & & & & & Cristobalite & $99.0 \pm 0.05$ & $0.8 \pm 0.06$ & $0.2 \pm 0.05$ \\
\hline & & & & & Metal & 0 & $0.21 \pm 0.02$ & $99.79 \pm 0.02$ \\
\hline \multirow[t]{2}{*}{27} & 1630 & $\mathrm{SiO}_{2} \mathrm{amp}$ & 1640 & $0.1+1$ & Liquid & $56.8 \pm 0.15$ & $12.7 \pm 0.18$ & $30.5 \pm 0.19$ \\
\hline & & & & & Cristobalite & $99.4 \pm 0.08$ & $0.2 \pm 0.05$ & $0.4 \pm 0.07$ \\
\hline \multirow[t]{3}{*}{28} & 1642 & $\mathrm{SiO}_{2} \mathrm{amp}$ & 1650 & $0.1+1$ & Liquid & $73.9 \pm 0.3$ & $14.8 \pm 0.2$ & $11.3 \pm 0.13$ \\
\hline & & & & & Cristobalite & $99.5 \pm 0.06$ & $0.3 \pm 0.03$ & $0.2 \pm 0.08$ \\
\hline & & & & & Metal & 0 & $0.23 \pm 0.02$ & $99.77 \pm 0.02$ \\
\hline \multirow[t]{2}{*}{29} & 1650 & $\mathrm{SiO}_{2} \mathrm{amp}$ & No & 0.7 & Liquid & $37.0 \pm 0.4$ & 0 & $63.0 \pm 0.4$ \\
\hline & & & & & Cristobalite & 99.7 & 0 & 0.3 \\
\hline \multirow[t]{3}{*}{30} & 1650 & $\mathrm{SiO}_{2} \mathrm{amp}$ & 1657 & $0.2+1$ & Liquid & $87.3 \pm 0.8$ & $5.3 \pm 0.3$ & $7.4 \pm 0.5$ \\
\hline & & & & & Cristobalite & $\sim 100$ & n.a. & \\
\hline & & & & & Metal & 0 & 0.4 & 99.6 \\
\hline \multirow[t]{3}{*}{31} & 1658 & $\mathrm{SiO}_{2} \mathrm{amp}$ & 1665 & $0.1+1$ & Liquid & $90.6 \pm 0.4$ & $6.6 \pm 0.3$ & $2.8 \pm 0.12$ \\
\hline & & & & & Cristobalite & $\sim 100$ & n.a. & \\
\hline & & & & & Metal & 0 & $0.4 \pm 0.16$ & $99.6 \pm 0.16$ \\
\hline \multirow[t]{2}{*}{32} & 1660 & $\mathrm{SiO}_{2} \mathrm{amp}$ & No & 0.7 & Liquid & $91.6 \pm 0.9$ & $1.9 \pm 0.12$ & $6.5 \pm 0.8$ \\
\hline & & & & & Cristobalite & $\sim 100$ & n.a. & \\
\hline \multirow[t]{2}{*}{33} & 1665 & $\mathrm{SiO}_{2} \mathrm{amp}$ & No & 0.7 & Liquid & $93.3 \pm 0.9$ & $4.7 \pm 0.6$ & $2.0 \pm 0.3$ \\
\hline & & & & & Cristobalite & $\sim 100$ & n.a. & \\
\hline \multirow[t]{3}{*}{34} & 1677 & $\mathrm{SiO}_{2} \mathrm{amp}$ & No & 0.5 & Liquid1 & $38.4 \pm 0.2$ & 0 & $61.6 \pm 0.2$ \\
\hline & & & & & Liquid2 & $95.1 \pm 0.1$ & 0 & $4.9 \pm 0.1$ \\
\hline & & & & & Cristobalite & 99.6 & 0 & 0.4 \\
\hline \multicolumn{9}{|c|}{2 liquids } \\
\hline \multirow[t]{2}{*}{35} & 1658 & $\mathrm{SiO}_{2} \mathrm{amp}$ & 1665 & $0.1+1$ & Liquid1 & $74.1 \pm 0.5$ & $18.9 \pm 0.4$ & $7.0 \pm 0.14$ \\
\hline & & & & & Liquid2 & $86.1 \pm 0.6$ & $9.9 \pm 0.5$ & $4.0 \pm 0.16$ \\
\hline 36 & 1660 & $\mathrm{SiO}_{2} \mathrm{amp}$ & No & 0.7 & Liquid1 & $55.4 \pm 1.8$ & $7.9 \pm 0.3$ & $36.7 \pm 1.6$ \\
\hline & & & & & Liquid2 & $79.6 \pm 0.5$ & $5.0 \pm 0.09$ & $15.4 \pm 0.4$ \\
\hline 37 & 1665 & $\mathrm{SiO}_{2} \mathrm{amp}$ & No & 0.7 & Liquid1 & $70.7 \pm 0.5$ & $21.4 \pm 0.4$ & $7.9 \pm 0.16$ \\
\hline & & & & & Liquid2 & $87.6 \pm 0.4$ & $8.8 \pm 0.3$ & $3.6 \pm 0.11$ \\
\hline Tridym & + cupr & oundary & & & & & & \\
\hline 38 & 1150 & $\mathrm{SiO}_{2} \mathrm{amp}$ & 1200 & $0.2+1+4$ & Liquid & $24.3 \pm 0.2$ & $8.5 \pm 0.2$ & $67.2 \pm 0.4$ \\
\hline & & & 1100 & & Tridymite & 99.8 & 0.1 & 0.1 \\
\hline & & & & & Cuprite & 0.0 & $0.1 \pm 0.01$ & $99.9 \pm 0.02$ \\
\hline 39 & 1160 & $\mathrm{SiO}_{2} \mathrm{amp}$ & 1200 & $0.2+1+4$ & Liquid & $22.1 \pm 0.4$ & $5.3 \pm 0.2$ & $73.8 \pm 0.5$ \\
\hline & & & 1100 & & Tridymite & 99.8 & 0.1 & 0.1 \\
\hline & & & & & Cuprite & 0.0 & $0.9 \pm 0.07$ & $99.9 \pm 0.07$ \\
\hline 40 & 1170 & $\mathrm{SiO}_{2} \mathrm{amp}$ & 1200 & $0.2+1+3$ & Liquid & $18.3 \pm 0.3$ & $4.0 \pm 0.1$ & $77.7 \pm 0.3$ \\
\hline & & & 1100 & & Tridymite & $99.7 \pm 0.15$ & $0.1 \pm 0.04$ & $0.2 \pm 0.11$ \\
\hline & & & & & Cuprite & $0.05 \pm 0.02$ & $0.15 \pm 0.03$ & $99.8 \pm 0.04$ \\
\hline Willem & + cupr & oundary & & & & & & \\
\hline 41 & 1160 & Pt-30\% Ir wire & 1165 & $0.2+2$ & Liquid & $23.1 \pm 0.2$ & $12.4 \pm 0.2$ & $64.5 \pm 0.3$ \\
\hline & & & & & Willemite & $33.3 \pm 0.11$ & $66.0 \pm 0.01$ & $0.7 \pm 0.11$ \\
\hline & & & & & Cuprite & 0.0 & $0.2 \pm 0.03$ & $99.8 \pm 0.03$ \\
\hline
\end{tabular}


Table 1 continued

\begin{tabular}{|c|c|c|c|c|c|c|c|c|}
\hline \multirow[t]{2}{*}{ No. } & \multirow[t]{2}{*}{$T,{ }^{\circ} \mathrm{C}$} & \multirow[t]{2}{*}{ Substrate } & \multirow[t]{2}{*}{ Premelt $T,{ }^{\circ} \mathrm{C}$} & \multirow[t]{2}{*}{ Time, $\mathrm{h}$} & \multirow[t]{2}{*}{ Phase } & \multicolumn{3}{|c|}{ Composition, mol.\% } \\
\hline & & & & & & $\mathrm{SiO}_{2}$ & $\mathrm{ZnO} / \mathrm{Zn}$ & $\mathrm{CuO}_{0.5} / \mathrm{Cu}$ \\
\hline \multicolumn{9}{|c|}{ Tridymite + willemite boundary } \\
\hline \multirow[t]{3}{*}{42} & 1140 & $\mathrm{SiO}_{2}$ amp + hole & 1240 & $0.25+2.5$ & Liquid & $26.8 \pm 0.3$ & $12.6 \pm 0.4$ & $60.6 \pm 0.6$ \\
\hline & & & & & Tridymite & $99.6 \pm 0.03$ & $0.36 \pm 0.4$ & $<0.1$ \\
\hline & & & & & Willemite & $32.6 \pm 0.14$ & $67.2 \pm 0.2$ & $0.22 \pm 0.16$ \\
\hline \multirow[t]{3}{*}{43} & 1150 & $\mathrm{Z}_{2} \mathrm{~S}^{*}+\mathrm{SiO}_{2}$ open crucible & 1240 & $0.25+2$ & Liquid & $28.2 \pm 0.3$ & $13.5 \pm 0.4$ & $58.3 \pm 0.6$ \\
\hline & & & & & Tridymite & 99.3 & 0.4 & 0.3 \\
\hline & & & & & Willemite & $33.1 \pm 0.16$ & $66.7 \pm 0.3$ & $0.24 \pm 0.3$ \\
\hline \multirow[t]{3}{*}{44} & 1200 & $\mathrm{SiO}_{2}$ open crucible & 1250 & $0.25+1.5$ & Liquid & $34.9 \pm 0.19$ & $20.2 \pm 0.3$ & $44.8 \pm 0.4$ \\
\hline & & & & & Tridymite & $99.3 \pm 0.1$ & $0.65 \pm 0.12$ & $0.08 \pm 0.02$ \\
\hline & & & & & Willemite & $33.4 \pm 0.06$ & $65.7 \pm 0.3$ & $0.8 \pm 0.3$ \\
\hline \multirow[t]{3}{*}{45} & 1250 & $\mathrm{Z}_{2} \mathrm{~S}+\mathrm{SiO}_{2}$ open crucible & 1260 & $0.25+1.5$ & Liquid & $39.3 \pm 0.2$ & $27.2 \pm 0.19$ & $33.5 \pm 0.3$ \\
\hline & & & & & Tridymite & $\sim 100$ & n.a. & n.a. \\
\hline & & & & & Willemite & $32.6 \pm 0.16$ & $66.9 \pm 0.2$ & $0.55 \pm 0.2$ \\
\hline \multirow[t]{3}{*}{46} & 1300 & $\mathrm{SiO}_{2}$ open crucible & 1320 & $0.25+1.5$ & Liquid & $42.7 \pm 0.14$ & $32.3 \pm 0.2$ & $25.0 \pm 0.2$ \\
\hline & & & & & Tridymite & $99.1 \pm 0.12$ & $0.84 \pm 0.1$ & $0.09 \pm 0.08$ \\
\hline & & & & & Willemite & $33.4 \pm 0.12$ & $66.1 \pm 0.12$ & $0.53 \pm 0.18$ \\
\hline \multirow[t]{3}{*}{47} & 1380 & $\mathrm{SiO}_{2} \mathrm{amp}$ & 1410 & $0.2+2$ & Liquid & $45.9 \pm 0.2$ & $44.2 \pm 0.18$ & $9.9 \pm 0.1$ \\
\hline & & & & & Tridymite & 98.8 & 1.1 & 0.1 \\
\hline & & & & & Willemite & $33.0 \pm 0.1$ & $66.8 \pm 0.12$ & $0.2 \pm 0.15$ \\
\hline \multicolumn{9}{|c|}{ Willemite $\left(\mathrm{Zn}_{2} \mathrm{SiO}_{4}\right)$ liquidus } \\
\hline \multirow[t]{2}{*}{48} & 1200 & Ir plate & 1240 & $0.25+1.5$ & Liquid & $11.0 \pm 0.4$ & $9.4 \pm 0.5$ & $79.6 \pm 0.8$ \\
\hline & & & & & Willemite & $33.0 \pm 0.18$ & $66.4 \pm 0.5$ & $0.65 \pm 0.6$ \\
\hline \multirow[t]{2}{*}{49} & 1250 & $\mathrm{Z}_{2} \mathrm{~S}+\mathrm{SiO}_{2}$ open crucible & 1260 & $0.25+1.5$ & Liquid & $36.9 \pm 0.3$ & $26.4 \pm 0.4$ & $36.7 \pm 0.5$ \\
\hline & & & & & Willemite & $32.6 \pm 0.16$ & $66.9 \pm 0.2$ & $0.55 \pm 0.2$ \\
\hline \multirow[t]{2}{*}{50} & 1250 & Pt-30\%Ir wire & 1280 & $0.2+1.5$ & Liquid & $16.3 \pm 0.3$ & $15.4 \pm 0.5$ & $68.3 \pm 0.7$ \\
\hline & & & & & Willemite & $33.0 \pm 0.08$ & $66.8 \pm 0.13$ & $0.18 \pm 0.09$ \\
\hline 51 & 1250 & Pt-30\%Ir wire & 1280 & $0.2+1.5$ & Liquid & $19.1 \pm 0.6$ & $17.6 \pm 0.5$ & $63.3 \pm 1.0$ \\
\hline & & & & & Willemite & $33.0 \pm 0.18$ & $66.7 \pm 0.3$ & $0.3 \pm 0.13$ \\
\hline 52 & 1250 & Pt-30\% Ir wire & 1280 & $0.2+1.5$ & Liquid & $25.4 \pm 0.3$ & $22.1 \pm 0.3$ & $52.5 \pm 0.5$ \\
\hline & & & & & Willemite & $32.9 \pm 0.06$ & $66.7 \pm 0.3$ & $0.45 \pm 0.3$ \\
\hline 53 & 1300 & Pt-30\%Ir wire & 1320 & $0.2+1$ & Liquid & $23.5 \pm 0.15$ & $28.7 \pm 0.3$ & $47.8 \pm 0.3$ \\
\hline & & & & & Willemite & $33.0 \pm 0.13$ & $66.7 \pm 0.3$ & $0.28 \pm 0.4$ \\
\hline 54 & 1300 & Pt-30\% Ir wire & 1320 & $0.2+1$ & Liquid & $31.3 \pm 0.13$ & $30.1 \pm 0.15$ & $38.5 \pm 0.2$ \\
\hline & & & & & Willemite & $32.9 \pm 0.12$ & $66.8 \pm 0.17$ & $0.34 \pm 0.19$ \\
\hline 55 & 1300 & Pt-30\%Ir wire & 1320 & $0.2+1$ & Liquid & $32.2 \pm 0.08$ & $30.8 \pm 0.11$ & $37.0 \pm 0.03$ \\
\hline & & & & & Willemite & $32.9 \pm 0.12$ & $66.8 \pm 0.17$ & $0.34 \pm 0.19$ \\
\hline 56 & 1350 & Pt-30\%Ir wire & No & 0.3 & Liquid & $21.1 \pm 0.09$ & $45.6 \pm 0.3$ & $33.3 \pm 0.3$ \\
\hline & & & & & Willemite & $33.2 \pm 0.09$ & $66.6 \pm 0.14$ & $0.2 \pm 0.18$ \\
\hline Willem & $\mathrm{Zn}_{2} \mathrm{SiC}$ & + zincite $(\mathrm{ZnO})$ boundary & & & & & & \\
\hline 57 & 1210 & Pt-30\%Ir wire & 1230 & $0.2+1$ & Liquid & $2.5 \pm 0.07$ & $7.8 \pm 0.2$ & $89.7 \pm 0.2$ \\
\hline & & & & & Willemite & $33.1 \pm 0.08$ & $66.2 \pm 0.3$ & $0.7 \pm 0.4$ \\
\hline & & & & & Zincite & $0.02 \pm 0.02$ & $98.1 \pm 0.04$ & $1.9 \pm 0.02$ \\
\hline 58 & 1250 & Pt-30\%Ir wire & 1300 & $0.25+1.5$ & Liquid & $4.8 \pm 0.13$ & $11.9 \pm 0.4$ & $83.3 \pm 0.5$ \\
\hline & & & & & Willemite & $33.1 \pm 0.11$ & $66.5 \pm 0.3$ & $0.45 \pm 0.2$ \\
\hline & & & & & Zincite & $0.07 \pm 0.03$ & $97.5 \pm 0.3$ & $2.4 \pm 0.3$ \\
\hline 59 & 1300 & Pt-30\% Ir wire & 1320 & $0.25+1.5$ & Liquid & $11.5 \pm 0.3$ & $26.0 \pm 0.5$ & $62.6 \pm 0.7$ \\
\hline & & & & & Willemite & $32.8 \pm 0.16$ & $67.0 \pm 0.5$ & $0.18 \pm 0.4$ \\
\hline & & & & & Zincite & $0.11 \pm 0.03$ & $97.9 \pm 0.18$ & $1.9 \pm 0.17$ \\
\hline 60 & 1330 & Pt-30\% Ir wire & No & 0.3 & Liquid & $16.3 \pm 0.3$ & $36.8 \pm 0.3$ & $46.9 \pm 0.6$ \\
\hline & & & & & Willemite & $33.1 \pm 0.12$ & $66.7 \pm 0.12$ & $0.2 \pm 0.14$ \\
\hline & & & & & Zincite & $0.15 \pm 0.03$ & $97.7 \pm 0.4$ & $2.1 \pm 0.4$ \\
\hline
\end{tabular}


Table 1 continued

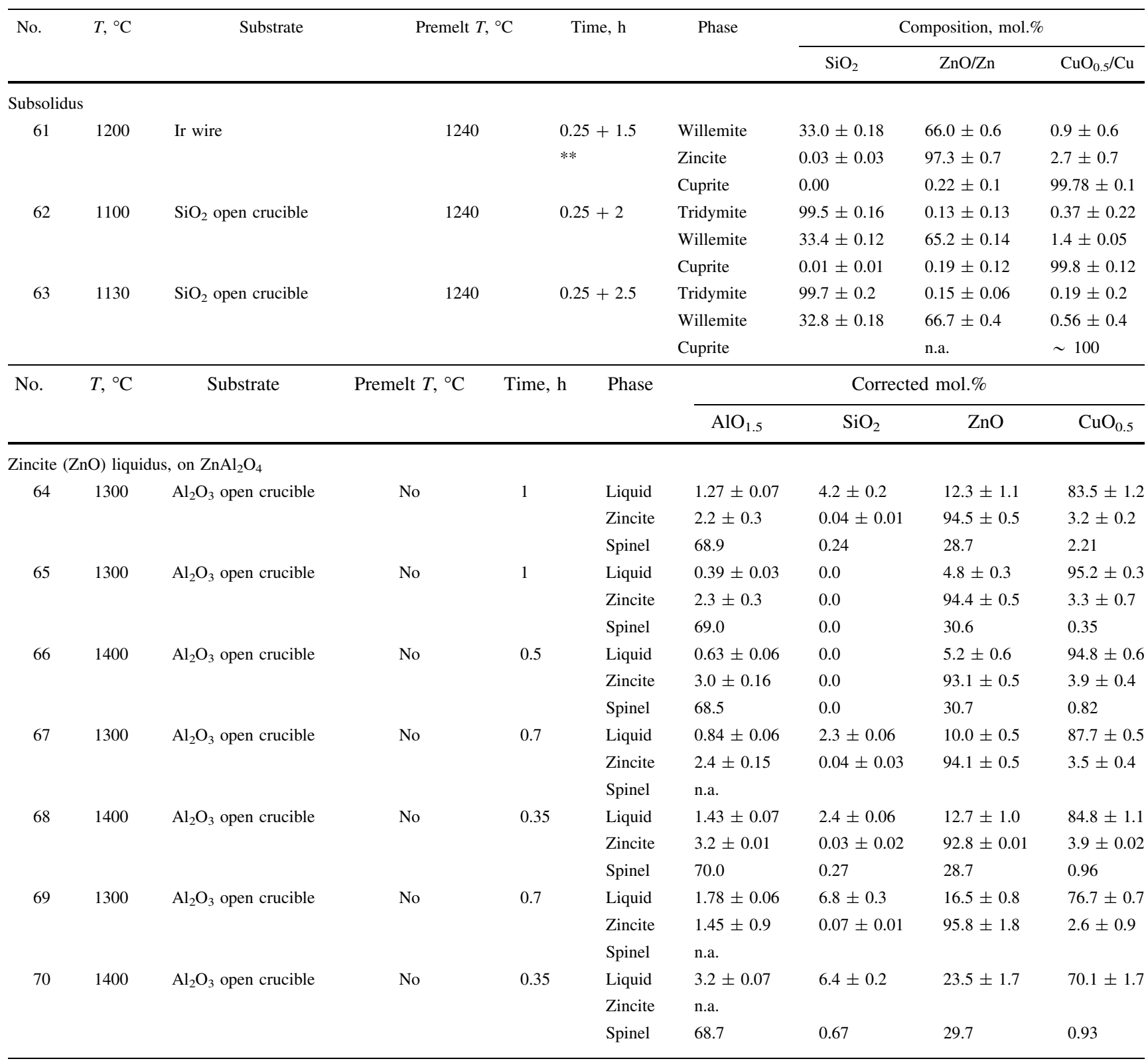

All listed samples also contained $\mathrm{Cu}$ metal. Its composition is given only when at least $0.1 \mathrm{~mol} \% \mathrm{Zn}$ in metal could be detected *Willemite $\left(\mathrm{Zn}_{2} \mathrm{SiO}_{4}\right)$-covered tridymite crucible

**All subsolidus experiments were premelted above solidus, so that $>50 \%$ liquid was expected to form. No remaining liquid slag was found, that means crystallization was complete. This fulfilled the main purpose of these experiments-bracketing the invariant temperatures from the below

is deduced from the steep zincite liquidus. This range is difficult to confirm due to high volatility of $\mathrm{Zn}$. Solubility of $\mathrm{ZnO}$ in $\mathrm{Cu}$-rich slag rapidly increases on addition of $\mathrm{SiO}_{2}$, and zincite primary phase field is replaced by willemite and later tridymite. The curvature of isotherms in the tridymite and cristobalite phase fields towards higher $\mathrm{SiO}_{2}$ in the central area of the triangle indicates strong negative interaction energy between $\mathrm{ZnO}$ and " $\mathrm{CuO}_{0.5}$ " in the silicate solution, opposite to what is observed in $\mathrm{SiO}_{2}$-free solutions. This stabilization of solutions along the $\mathrm{ZnO} \cdot \mathrm{CuO}_{0.5}-\mathrm{SiO}_{2}$ line becomes so significant above $1600{ }^{\circ} \mathrm{C}$ that the areas of two liquids in the " $\mathrm{CuO}_{0.5}$ " $-\mathrm{SiO}_{2}$ and $\mathrm{ZnO}-\mathrm{SiO}_{2}$ binaries disappear towards the $1: 1 \mathrm{Cu}: \mathrm{Zn}$ ratio. This makes the present system an example of importance to study ternary systems in wide ranges of compositions, as their properties cannot be accurately 
Fig. 2 Liquidus points of the $\mathrm{ZnO}-“ \mathrm{CuO}_{0.5}$ "- $\mathrm{SiO}_{2}$ system in equilibrium with metal, compared to literature data by Xia et al. ${ }^{[2]}$ and Hidayat ${ }^{[17]}$.

(a) complete diagram,

(b) zoomed $\mathrm{Cu}$-rich area. Points on " $\mathrm{CuO}_{0.5}$ "- $-\mathrm{SiO}_{2}$ and $\mathrm{ZnO}$ $\mathrm{SiO}_{2}$ binaries have been also reported in $\operatorname{Ref}{ }^{[14,16]}$. Isotherms and boundaries are drawn using the present experimental points (where available) or estimated with a preliminary thermodynamic model. *There is no direct experimental confirmation for two liquids existence in the $\mathrm{ZnO}$ - " $\mathrm{CuO}_{0.5}$ " system, it is suggested based on extrapolation of lowtemperature data

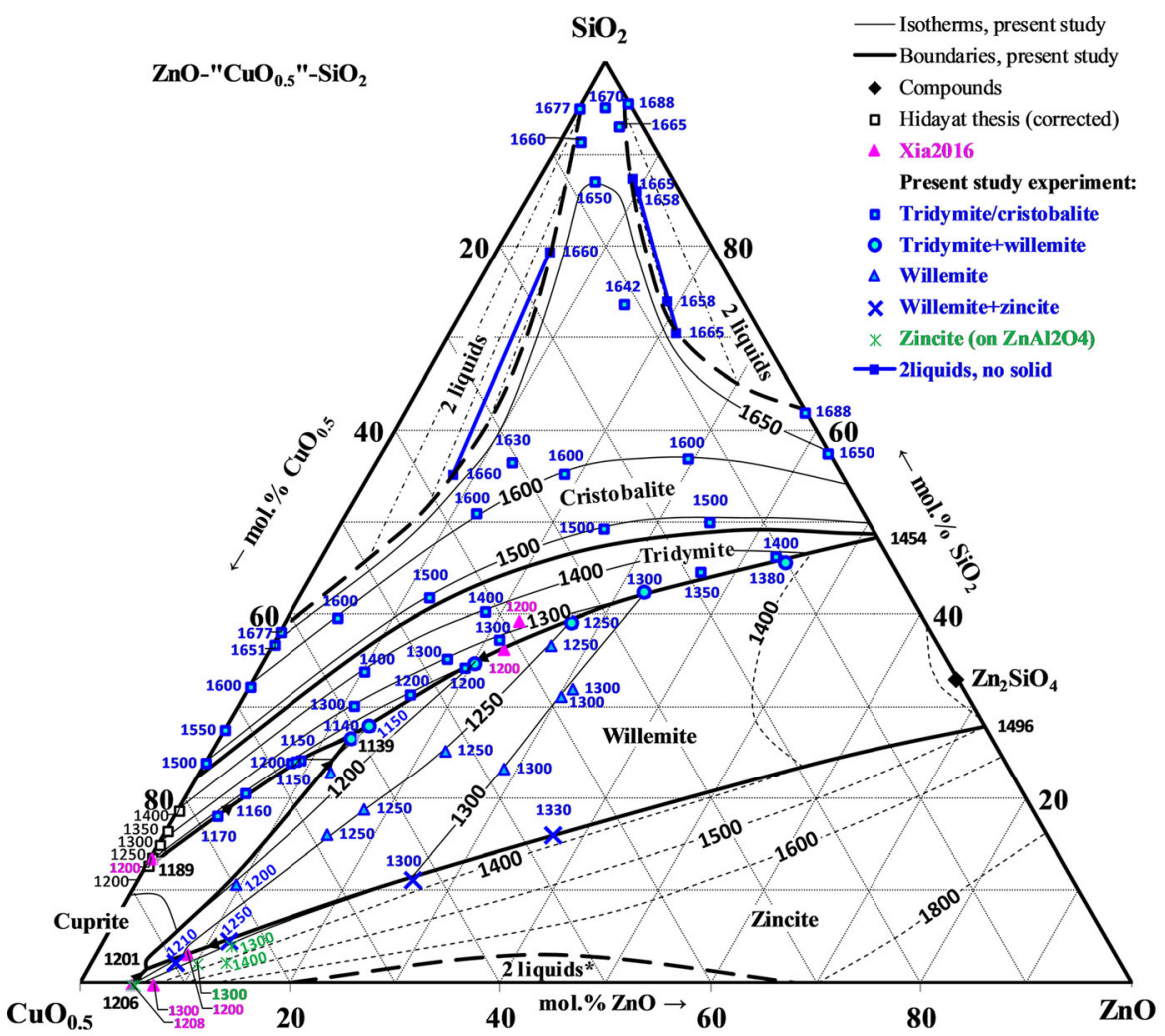

(a)

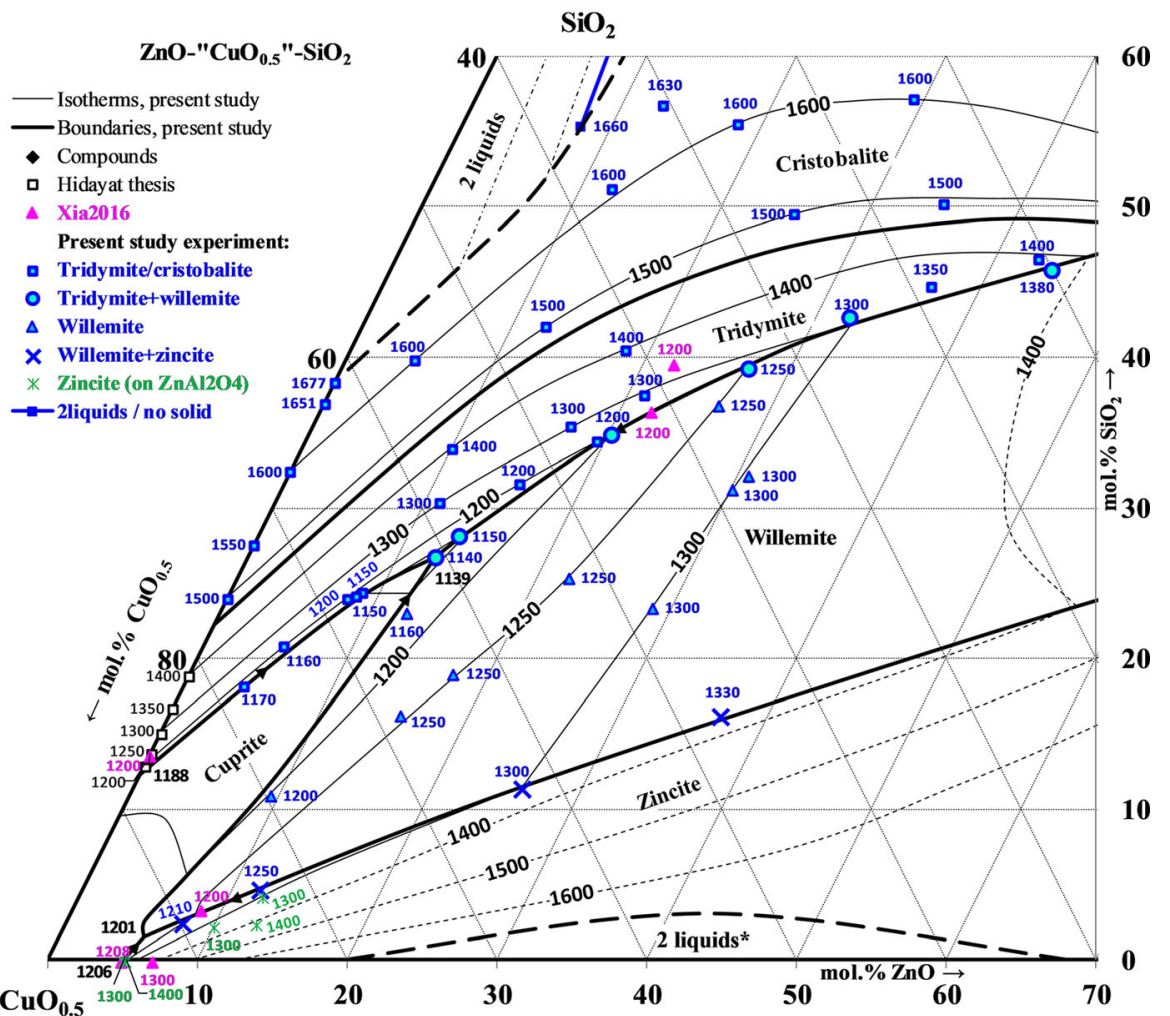

(b) 
Fig. 3 Liquidus points of the $\mathrm{ZnO}-$ " $\mathrm{CuO}_{0.5}$ " system in equilibrium with metal, compared to literature data by $\mathrm{Xia}$ et al. ${ }^{[2]} *$ There is no direct experimental confirmation for two liquids existence in the $\mathrm{ZnO}-$ "CuO 0.5 " system, it is suggested based on extrapolation of lowtemperature data

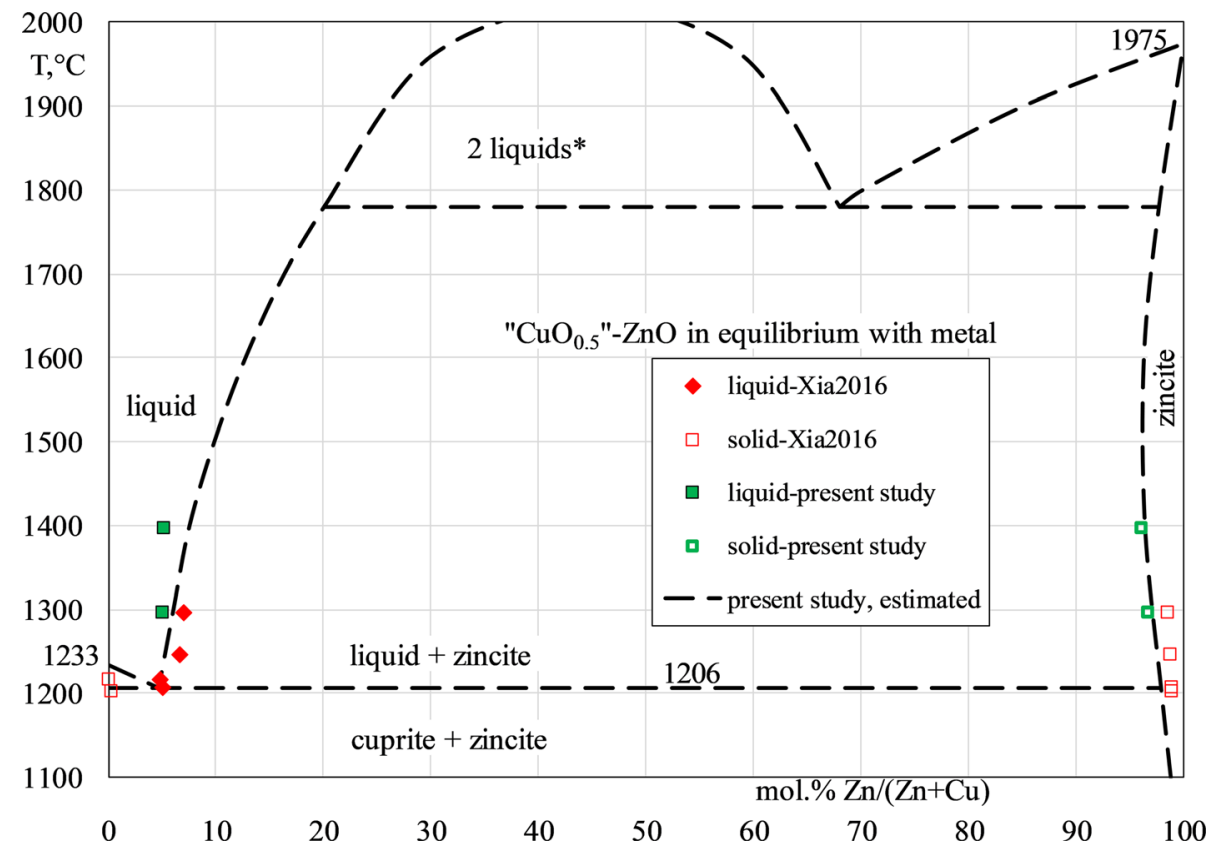

predicted from the limiting binaries-in this case, three systems with positive interactions combine into a ternary with negative interactions, and miscibility gaps disappear.

The combination of cristobalite and two immiscible liquids exists in many ternary systems over a range of temperatures. It is not prohibited by Gibbs rule. Unlike the binary system, a small change in temperature does not destroy such phase assemblage: a new equilibrium will be established on another tieline between two immiscible liquids, with change in proportion of two liquids and cristobalite. Examples of this phase assemblage were recently published by the authors. ${ }^{[14,20]}$ There is no need to assume constant diffusion of $\mathrm{SiO}_{2}$. Moreover, the experiments in the manuscript were indeed measured at variable distances from the substrate (30-500 micron), and no significant variation in compositions of liquids were detected.

In the $\mathrm{CuO}_{0.5}-\mathrm{SiO}_{2}$ and $\mathrm{ZnO}-\mathrm{SiO}_{2}$ binaries, the structures corresponding to monotectic temperatures $\left(1677{ }^{\circ} \mathrm{C}\right.$ and $1688{ }^{\circ} \mathrm{C}$, respectively) are metastable and one phase is expected to disappear within much longer time (that was unpractical to use due to high failure risk of the furnace and substrate at these high temperatures). The gradients in composition of the phases were within only $0.3 \%\left(\mathrm{CuO}_{0.5^{-}}\right.$ $\left.\mathrm{SiO}_{2}\right)$ and $0.6 \%\left(\mathrm{ZnO}-\mathrm{SiO}_{2}\right)$. This indicates that the real monotectic temperatures are very close to the experimental temperatures (possible $5{ }^{\circ} \mathrm{C}$ uncertainty including the uncertainty of the furnace controller and thermocouple).

\section{Conclusions}

Phase equilibria of the $\mathrm{ZnO}$ - " $\mathrm{CuO}_{0.5}$ " and $\mathrm{ZnO}$ - " $\mathrm{CuO}_{0.5}$ "$\mathrm{SiO}_{2}$ systems in equilibrium with $\mathrm{Cu}-\mathrm{Zn}$ metal at liquidus have been investigated at $1403-1948 \mathrm{~K}\left(1130-1675^{\circ} \mathrm{C}\right)$. Tridymite, cristobalite, willemite, zincite, cuprite and two immiscible liquids fields have been identified in the wide range of compositions. The obtained results will be used for development of multicomponent thermodynamic database for copper, lead and zinc pyrometallurgy.

Acknowledgments The authors would like to thank Nyrstar (Australia), Outotec Pty Ltd (Australia), Aurubis AG (Germany), Umicore NV (Belgium), and Kazzinc Ltd, Glencore (Kazakhstan), and Australian Research Council Linkage project LP150100783 for their financial support for this research. The authors are grateful to Prof. Peter C. Hayes (UQ) for valuable comments and suggestions, to Ms. Suping Huang, Mr. Shuyi Lou, Mr. Ryan Wright for assistance with conducting experiments, and to the staff of the University of Queensland Centre for Microanalysis and Microscopy (CMM) for their support in maintenance and operation of scanning and electron microprobe facilities in the Centre.

\section{References}

1. L. Xia, Z. Liu, and P.A. Taskinen, Phase Equilibria Study of $\mathrm{Cu}-$ $\mathrm{O}-\mathrm{ZnO}$ System in Various Oxygen Partial Pressures, Ceram. Int., 2016, 42(4), p 5418-5426 
2. L. Xia, Z. Liu, P.A. Taskinen, Experimental Determination of the Liquidus Surface (1473 k) in $\mathrm{Cu}-\mathrm{ZnO}-\mathrm{SiO}_{2}-\mathrm{O}$ System at Various Oxygen Partial Pressures, in Advances in Molten Slags, Fluxes, and Salts: Proceedings of the 10th International Conference on Molten Slags, Fluxes and Salts (MOLTEN16), TMS (The Minerals, Metals \& Materials Society, 2016), pp 971-978

3. L. Xia, Z. Liu, and P. Taskinen, Experimental Determination of the Liquid Phase Domain of the $\mathrm{Cu}-\mathrm{O}-\mathrm{ZnO}-\mathrm{SiO}_{2}$ System in Equilibrium with Air, Can. Metall. Q., 2017, 56(1), p 10-17

4. T. Hidayat, H.M. Henao, P.C. Hayes, and E. Jak, Phase Equilibria Studies of the $\mathrm{Cu}-\mathrm{Fe}-\mathrm{O}-\mathrm{Si}$ System in Equilibrium with Air and with Metallic Copper, Metall. Mater. Trans. B, 2012, 43, p 10341045

5. E. Jak, P.C. Hayes, and H.-G. Lee, Improved Methodologies for the Determination of High Temperature Phase Equilibria, Korean J. Miner. Mater. Inst. (Seoul), 1995, 1(1), p 1-8

6. E. Jak, Integrated Experimental and Thermodynamic Modelling Research Methodology for Metallurgical Slags with Examples in the Copper Production Field, in 9th International Conference on Molten Slags, Fluxes and Salts (MOLTEN12), W077 (The Chinese Society for Metals, Beijing, China, 2012).

7. C. Landolt, Equilibrium Studies in the System Copper-SiliconOxygen, Ph.D. Thesis, Pennsylvania State University, 1969

8. J. Philibert, Method for Calculating the Absorption Correction in Electron-probe Microanalysis, in 3rd International Symposium on $X$-ray Optics and X-ray Microanalysis (Stanford University, 1963), pp. 379-392

9. P. Duncumb, S.J.B. Reed, Calculation of Stopping Power and Backscatter Effects in Electron Probe Microanalysis (1968)

10. P. Duncumb, Quantitative Electron Probe Microanalysis, in 25th Anniversary Meeting of the Electronmicroscopy and Analysis (1971), pp. 132-137

11. M. Shevchenko and E. Jak, Experimental Liquidus Studies of the $\mathrm{Pb}-\mathrm{Fe}-\mathrm{Si}-\mathrm{O}$ System in Equilibrium with Metallic $\mathrm{Pb}$, Metall. Mater. Trans. B, 2018, 49(1), p 159-180
12. M. Shevchenko and E. Jak, Experimental Phase Equilibria Studies of the PbO-SiO 2 System, J. Am. Ceram. Soc., 2017, 101(1), p 458-471

13. M. Shevchenko, J. Chen, E. Jak, Establishing Additional Correction for Quantitative EPMA Measurements in the System $\mathrm{PbO}-\mathrm{SiO}_{2}$, in AMAS 2017, 14th Biennial Australian Microbeam Analysis Symposium, 6-10 February (Brisbane, QUT, Australia, 2017), pp. 94-95

14. M. Shevchenko and E. Jak, Experimental Liquidus Studies of the Binary $\mathrm{Pb}-\mathrm{Cu}-\mathrm{O}$ and Ternary $\mathrm{Pb}-\mathrm{Cu}-\mathrm{Si}-\mathrm{O}$ Systems in Equilibrium with Metallic $\mathrm{Pb}-\mathrm{Cu}$ Alloys, J. Phase Equilib. Diffus., 2019, 40(5), p 671-685

15. M. Shevchenko and E. Jak, Experimental Liquidus Study of the Binary $\mathrm{PbO}-\mathrm{ZnO}$ and Ternary $\mathrm{PbO}-\mathrm{ZnO}-\mathrm{SiO}_{2}$ Systems, Ceram. Int., 2019, 45(6), p 6795-6803

16. M. Shevchenko and E. Jak, Experimental Liquidus Study of the Ternary CaO-ZnO- $\mathrm{SiO}_{2}$ System, Metall. Mater. Trans. B, 2018, 50(6), p 2780-2793

17. T. Hidayat, Equilibria Study of Complex Silicate-Based Slag in the Copper Production, $\mathrm{PhD}$ thesis, The University of Queensland, 2013

18. S. Nikolic, P.C. Hayes, and E. Jak, Experimental Techniques for Investigating Calcium Ferrite Slags at Metallic Copper Saturation and Application to the Systems " $\mathrm{Cu}_{2} \mathrm{O}$ " $-\mathrm{Fe}_{2} \mathrm{O}_{3}$ " and " $\mathrm{Cu}_{2} \mathrm{O}$ "$\mathrm{CaO}$ at Metallic Copper Saturation, Metall. Mater. Trans. B, 2009, 40B(6), p 892-899

19. M. Shevchenko and E. Jak, Experimental Liquidus Studies of the $\mathrm{Zn}-\mathrm{Fe}-\mathrm{Si}-\mathrm{O}$ System in Air, Int. J. Mater. Res. (IJMR), 2019, 110(7), p 600-607

20. M. Shevchenko and E. Jak, Experimental Liquidus Studies of the $\mathrm{Pb}-\mathrm{Fe}-\mathrm{Si}-\mathrm{O}$ System in Air, J. Phase Equilib. Diffus., 2019, 40(3), p 319-355

Publisher's Note Springer Nature remains neutral with regard to jurisdictional claims in published maps and institutional affiliations. 